\title{
Aortic Stenosis in the Elderly: Understanding the New Therapeutic Options
}

\author{
Valentina Boasi $^{{ }^{*}}$, Maria Carla Casale ${ }^{{ }^{*}}$, Milena Aste ${ }^{1}$, Giuseppe Tarantini² Sabina Gallina ${ }^{3}$, \\ Manrico Balbi ${ }^{1}$, Claudio Brunelli ${ }^{1}$, Gian Paolo Bezante ${ }^{1 \#}$ \\ ${ }^{1}$ Gian Paolo Bezante on Behalf of the Valvular Disease Working Group of the Italian Society of Cardiology (SIC), \\ Department of Internal Medicine, University of Genova Viale Benedetto, Genova, Italy \\ ${ }^{2}$ Clinica Cardiologica, University of Padova, Padova, Italy \\ ${ }^{3}$ Department of Cardiology, University G D'Annunzio, Chieti, Italy \\ Email: \#gian.paolo.bezante@unige.it
}

Received August 19, 2012; revised September 17, 2012; accepted September 26, 2012

\begin{abstract}
Aortic Stenosis (AS) has become the most frequent valvular heart disease in the western countries with increased prevalence in the elderly. It is associated with very poor prognosis in the absence of surgical treatment and worse quality of life. Patients aged between 80 - 85 years with no major comorbidities and left ventricular ejection fraction between $30 \%$ $-50 \%$ are still not referred to valve surgery, even if these subjects would likely benefit from surgery in terms of duration and quality of life as compared with the expected outcome of the disease. The decision making process in octogenarian population are not only related to the decision of whether to operate or not, but also to the timing of surgery. The identification of symptoms related to AS is difficult in the elderly and this also delay surgery. Balloon Aortic Valvuloplasty (BAV) and, in the recent years, Transcatheter Aortic Valve Implantation (TAVI) seem to offer an additional chance of valve correction, in patients at high surgical risk or inoperable. For ethical reasons, the first TAVI cases were performed exclusively on patients who had contraindications to surgery because of end stage heart disease and/or severe comorbidities. This accounted for high mid-term mortality rates; however, these findings demonstrated the feasibility of this alternative approach. An overall patient evaluation based on a team approach (involving cardiologists, surgeons and geriatricians) is essential. Results from randomized clinical trials on elderly patients who underwent TAVI are encouraging and result from one and two years follow-up are now available.
\end{abstract}

Keywords: Aortic Stenosis; Elderly; Transcatheter Aortic Valve Implantation (TAVI)

\section{Introduction}

Aortic Stenosis (AS) has become the most frequent valvular heart disease in the western world with an increasing prevalence in the elderly, while in older subjects it is a major cause of morbidity. When we say "old", we have to stress that the ageing process is highly individual and even if the age of 65 is frequently used as the indicator of the beginning of old age, the biological or physiological age of an individual is much more important. It provides us with a more accurate evaluation of the patient's "fragility" and how he/she may react to therapy. The lower limit of senescence is seventy years of age since the incidence of changes correlated to ageing increases strongly between seventy and seventy-five. Eighty-five years of age may be considered the borderline age for senescence if we keep in mind that the clinical fragility of most individuals is related to some form of organic dysfunction

\footnotetext{
${ }^{*}$ Contributed equally to this work
}

\#Corresponding author. (dementia affects more than $50 \%$ of people over eightyfive years of age) [1-3].

\subsection{Epidemiology and Aetiology in the Elderly}

The Cardiovascular Health Study, which involved 5201 men and women older than 65 years of age, showed that $26 \%$ of the participants had aortic sclerosis (a thickening of the valve or calcification without significant obstruction). A clear rise in the prevalence of sclerosis and stenosis was seen with increasing age: $20 \%$ in patients aged $65-75$ years, $35 \%$ in those aged $75-85$ years and $48 \%$ in those older than 85 years, whereas severe aortic stenosis increased by $1 \%-3 \%, 2 \%-4 \%$ and $4 \%$, respectively [4]. Aronow et al. observed that AS, as diagnosed by Doppler-echocardiography, was present in 141 of 924 men $(15 \%)$, mean age 80 , and in 322 of 1881 women $(17 \%)$, mean age 81 . In a cohort of 2805 elderly patients, severe AS was diagnosed in 62 cases (2\%), moderate AS was present in $149(5 \%)$, and mild AS occurred in 25 
$(9 \%)[5]$.

With the decline of acute rheumatic fever, calcific aortic stenosis has become the most common indication for valvular disorder in thewestern countries, especially in adults of advanced age $(2 \%-7 \%$ of the population $>65$ years), (Figure 1) [6-8]. Recent descriptive studies from patient specimens have demonstrated the hallmark features of aortic valve disease: including early atherosclerosis, cell proliferation and osteoblast expression. To understand aortic valve disease, three interrelated events responsible for the development of valve calcification to consider: classical cardiovascular risk factors, genetic factors and valve biology. The interrelationship of these three pathways leading to the development of human aortic valve calcification can be found in many experimental and clinical studies as reported by Rajamannan [9]. In the past decade, large epidemiologic cohort studies showed as the traditional risk factors for cardiovascular atherosclerosis (such as lipids, hypertension, male gender, renal failure, and diabetes) were also important in the development of calcific aortic stenosis.

The role of lipids as a risk factor for vascular atherosclerosis has been defined in the literature for years. The understandings of these clinical risk factors are providing the foundation for the cellular studies and the potential for targeted medical therapies for this disease similar to vascular atherosclerosis. The discovery of atherosclerosis in the aortic valve in patients with familial hypercholesterolemia provides the initial proof for potential treatment with lipid-lowering drugs to control the progression of aortic valve disease. Recent clinical trials don't confirm it and anyway mechanism of the effect of statins on the disease process in the aortic valve remains unclear. [10]

In addition, Angiotensin-Converting Enzyme (ACE) and angiotensin II type 1 and type 2 receptors are present in aortic stenosis, implicating this signaling pathway in the disease process. The hemodynamic effects of ACE inhibitors might obscure the effects at the tissue level by changing the flow conditions across the valve [11].

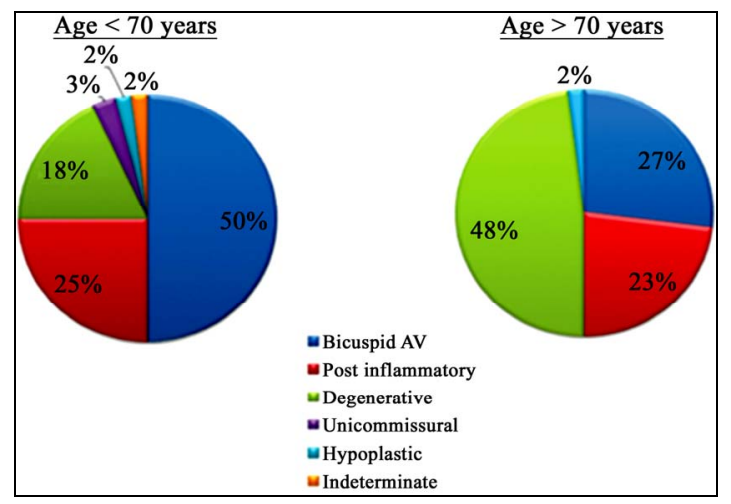

Figure 1. Differences in aetiology of aortic stenosis based on a cut-off age of 70 years.
In conclusion, despite many studies in this direction, actually there is no medical therapy able to slow disease progression, even more in the elderly, where disease is more represented daily.

\subsection{Physiology}

AS is defined as an obstacle to the blood flow through the aortic valve in the systolic ejection of the Left Ventricle (LV). This obstacle in LV outflow leads to increases in both systolic pressure and in diastolic LV pressure, prolonged ejection time, and a decrease in aortic pressure, thus establishing a trans-valve gradient $[12,13]$. These alterations occur when the aortic area is reduced to at least $50 \%$. The pressure overload is compensated by the myocardial hypertrophy, without LV enlargement (concentric hypertrophy) and so normal systolic function may be preserved for many years. Increased systolic pressure, ventricular mass and ejection time all result in higher oxygen consumption in the myocardium. Prolonged outflow time will result in a reduction in diastolic time, an increase in diastolic pressure and a decrease in aortic pressure. This will lead to a decrease in coronary perfusion and the consequent reduction of oxygen supply to the cardiomyocytes. Themyocardial ischemia that occurs due to these alterations will further worsen LV function. In a more advanced phase, disease progression will produce a mismatch between ventricular pump function and afterload (afterload mismatch). Hence, we observe ventricular chamber enlargement, reduced cardiac output and ejection fraction, and increases in both diastolic filling pressure and pulmonary pressure. This phase generally coincides with symptoms that usually begin around the sixth decade of life after a long period of latency, and is characterized by progressive thickening and calcification of a congenital or rheumatic aortic valve and/or progressive myocardial dysfunction. In the 1960s, the first preload reserve and afterload mismatch studies were carried out. In 1964, Ross and Braunwald documented how the $\mathrm{LV}$ potential reserve is exhausted as the afterload increases. In later laboratory studies on animals, preload, inotropism and cardiac frequency were kept constant and afterload changes alone were induced. In this setting, sudden afterload changes in the normal heart reduced stroke volume and vice-versa [14]. Other studies on healthy hearts confirmed the inverse relationship between afterload and stroke volume when the preload is kept unchanged. Preload variations on the other hand can change this correlation. When afterload increases, and if preload also increases to compensate, stroke volume will not vary substantially. When the preload reserve is surpassed, thus causing heavily elevated ventricular filling pressures, any further afterload increase will result in a reduction in the stroke volume. Afterload mismatch is the inability of the LV to maintain adequate stroke volume as 
the afterload increases despite a preload increase. In severe AS, when ejection fraction diminishes, the LV preload reserve is even more limited by cardiac hypertrophy [15]. As the diameter of the valvular orifice decreases, the LV afterload further increases with a net reduction of ejection fraction, mainly due to the absence of preload reserve. If the patient undergoes AVR, ejection fraction will improve remarkably because of the net reduction of afterload. However, this only occurs if the myocardium is not overly impaired prior to surgery. As a consequence, the timing decision for valve replacement is crucial: distinguishing between impaired LV function due to afterload mismatch or due to irreversible myocardial damage is difficult in basal conditions. When LV function begins to diminish, the pressure-volume curve shows an increase in both the end-diastolic volume (preload reserve) and the systolic wall stress. In this first phase, the ejection fraction is preserved. As the AS worsens, afterload increases, preload reserve is worn out, and cardiac stroke and ejection fraction are remarkably reduced $[16,17]$.

\subsection{Natural History}

AS is a progressive disease and its severity increases with time. Over a long, latent period, patients remain asymptomatic but the duration of the asymptomatic phase varies widely among individuals. The elderly also have different, often more complicated health care problems, such as multiple disorders, which may require use of many drugs (polypharmacy). Diagnosis may be complicated, resulting in delays or missed diagnoses, and sometimes drugs are used inappropriately. Early detection of problems results in early intervention, which can prevent deterioration and improve quality of life often through relatively minor, inexpensive interventions (e.g. lifestyle changes).

\subsubsection{Clinical Features}

Early features may be relatively non-specific, such as palpitations, dizziness and fatigue, and some people may be unaware of a gradual decline in their exercise capacity. It is therefore important to be aware of the possibility of aortic stenosis, and we suggest that auscultation at the apex and right parasternal position should be performed in every patient aged over 70 years who presents with such symptoms. As soon as symptoms occur, survival becomes poor and mortality has been reported to be quite significant, occurring even within months from symptom onset [18]. In particular, the average survival rate after the onset of angina pectoris was estimated at 5 years in patients with severe AS, while after the onset of syncope it was 3 years, and the average survival rate after the onset of congestive heart failure was 1.5 - 2 years (Figure 2) [19]. In 4\% of the patients with aortic stenosis, the first symptom issudden death, usually during strenuous exertion. Patients with symptomatic, severe AS have a poor prognosis. Data from the National Institutes of Health reveal that $52 \%$ of patients with symptomatic, severe AS who did not undergo surgery died within 5 years, and that at 10 -year follow-up, $90 \%$ of these patients were decreased. Moreover, patients with severe AS and pulmonary hypertension who do not undergo Aortic Valve Replacement (AVR) have an $80 \%$ mortality rate at a median follow-up of 436 days [20,21].

\subsubsection{Asymptomatic Patients}

Studies have shown that patients with severe, asymptomatic AS are at low risk of sudden cardiac death (approx. 1\%/year) [22,23]. Pellikka et al. followed-up 622 patients with asymptomatic AS and peak systolic velocity $\geq 4$ $\mathrm{m} / \mathrm{sec}$. Patients who did not undergo surgery had probability of remaining cardiac-symptom free of $82 \%, 67 \%$, and $33 \%$ at 1,2 , and 5 years, respectively. These authors found that aortic valve area and left ventricular hypertrophy predicted symptom development, and in fact, patients with peak aortic jet velocity $\geq 4.5 \mathrm{~m} / \mathrm{sec}$ had a greater likelihood of developing symptoms or having surgery or cardiac death [22]. These data were confirmed by Rosenhek, R. et al. who followed-up 116 asymptomatic patients with very severe, isolated aortic stenosis (defined by a peak aortic jet velocity $\geq 5.0 \mathrm{~m} / \mathrm{sec}$ ). Despite being asymptomatic, these patients had a poor prognosis, a high event rate (Event-free survival was 64\%, 36\%, 25\%, 12\%, and $3 \%$ at $1,2,3,4$, and 6 years, respectively), and a risk of rapid functional deterioration. Early elective valve replacement surgery was therefore taken into consideration for these patients [24]. In the population-based KORA/ MONICA survey, age andbaseline status of smoking and total cholesterol levels were predictive of significant AS [25].

\subsubsection{Identification of High Risk Patients}

The following predictors of the progression of AS and/or of poor outcome in asymptomatic patients were recently proposed:

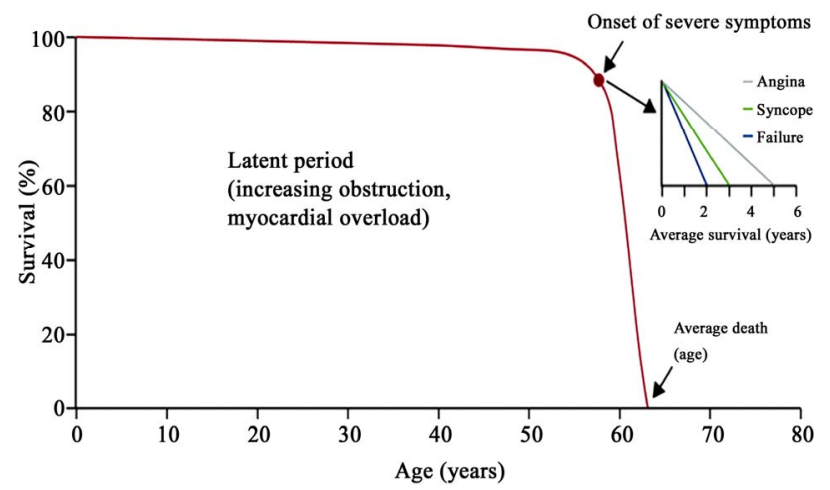

Figure 2. The natural history of aortic stenosis: average survival after onset of symptoms. 
- Clinical factors (older age, presence of atherosclerotic risk factors). Peltier et al. analysed the relationship between cardiovascular risk factors and nonrheumatic, calcific AS in 220 patients (mean age $68 \pm$ 9 years). They found that total cholesterol $(>200$ $\mathrm{mg} / \mathrm{dl})$, BMI $\left(>30 \mathrm{~kg} / \mathrm{m}^{2}\right)$, history of smoking and systemic hypertension were all independent markers of AS [26].

- Echocardiographic data (valve calcification, peak aortic jet velocity, LVEF, haemodynamic progression and increase in gradient with exercise). The combination of a markedly calcified valve and a rapid increase in velocity of $0.3 \mathrm{~m} / \mathrm{sec}$ within 1 year has been shown to identify a high-risk group of patients $(80 \%$ death or need for surgery within 2 years) [27].

- Results from exercise testing: Symptom development during exercise testing in physically active patients (particularly those younger than 70 years of age) predicts a very high likelihood of symptom development within 12 months [28]. Recent data-demonstrate a lower positive predictive value for abnormal blood pressure response, and even more so for ST-segment depression, than symptoms for poor outcome.

\section{Diagnosis: Role of Cardiac Imaging}

Aortic stenosis in elderly people may be without clinical signs. Echocardiography has become the key tool for the diagnosis and evaluation of valve disease, and is the primary non-invasive imaging method for valve stenosis assessment. Routine echocardiography increases the sensitivity of the clinical diagnosis, and may have major therapeutic implications for the elderly patient. The current American College of Cardiology/American Heart Association guidelines for the management of valvular heart disease recommend echocardiography for "holo-systolic or late systolic" and "grade 3 or greater mid-systolic" murmurs [28].

M-mode and 2-dimensional echocardiography can detect thickening, calcification, and a reduced excursion of aortic valve leaflets, they can also reveal the presence of LV hypertrophy, measure chamber size and LV endsystolic and end-diastolic volume, and assess LV ejection fraction and global and regional LV wall motion [29].

Doppler echocardiography is used to measure peak and mean gradients across the aortic valve and to identify associated valve lesions. Aortic valve area can be calculated by the continuity equation using pulsed Doppler echocardiography to measure LV outflow tract velocity, continuous-wave Doppler echocardiography to measure flow velocity, and 2 dimensional long-axis views to measure LV outflow tract area [30-32].

By matching data from valvular area and pressure gradients it is possible to definesevere AS when peak Doppler velocity is greater than $4 \mathrm{~m} / \mathrm{sec}$ and the mean pres- sure gradient is greater than $40 \mathrm{mmHg}$ according to the American guidelines, or $50 \mathrm{mmHg}$ according to the European guidelines [28]. With regard to clinical decisionmaking, valve area should be considered together with flow rate, pressure gradient and ventricular function, as well as with functional status. Although AS with a valve area $<1.0 \mathrm{~cm}^{2}$ is generally considered severe, indexing to Body Surface Area (BSA), with a cut-off value of 0.6 $\mathrm{cm}^{2} / \mathrm{m}^{2}$ BSA is especially helpful in patients with either unusually small or large BSA; the elderly patient is sometime cachectic and has a very small BSA [33].

Low-pressure gradients may be observed in patients with severe AS and depressed LV function. As soon as the mean gradient is $40 \mathrm{mmHg}$, even a small valve area does not definitelyconfirm severe AS since mild-to-moderately diseased valves may not open fully, thus resulting in a "functionally small valve area (pseudo-severe AS)" [28].

In this setting, stress echocardiography using low-dose dobutamine (DOB) may be helpful to distinguish truly severe AS from the rare cases of pseudo-severe AS. Truly severe AS shows only small changes in valve area (increase $<0.2 \mathrm{~cm}^{2}$ ) with increasing flow rate but significant increases in gradients (maximum value of mean gradient $>50 \mathrm{mmHg}$ ), whereas pseudo-severe AS shows marked increases in valve area but only minor changes in gradients. Moreover, DOB stress-echo may detect the presence of contractile reserve, defined as an increase $(>20 \%)$ of stroke volume during low-dose DOB infusion, which has important prognostic implications [34]. Echocardiographic evaluation can identify co-existing valvular lesions such as mitral annular calcifications in degenerative disease and rheumatic mitral valve disease, as well as asymmetric dynamic sub-aortic obstruction especially in elderly women. Doppler estimation of pulmonary systolic pressure completes the data for the therapeutic and prognostic implications.

Transesophageal echocardiogram is rarely needed, however, it can be useful to allow valve planimetry especially when transthoracic visualization is poor. In the TAVI (Transcatheter Aortic Valve Implantation) Era, precise annulus and aortic root measurements is fundamental. Knowing the exact position of the coronary ostii and their precise distance from the annulus is essential during TAVI procedures [32].

Computed Tomography (CT) can provide a quantitative assessment of valve calcification and has been shown to correlate with echocardiographic assessment and clinical outcome. The role of CT in clinical management is not yet well defined, butmay facilitate the selection of candidates for transcatheter aortic valve. In TAVI patients multi slice CT can provide detailed information on the shape of the aortic annulus, that is commonly eccentric and often oval, and the relation be- 
tween the annulus and the ostium of the coronary arteries. In these patients $\mathrm{CT}$ is also used to assess calcification and tortuosity of femoroiliac vascular axes [35].

Due to the high accuracy of ultrasound assessment of AS severity, cardiac catheterization is currently devoted to exclude the presence of associated coronary artery disease.

\section{Traditional Surgery and New Therapeutic Options}

\subsection{Traditional Aortic Valve Replacement}

The only effective treatment in adult patients with severe symptomatic calcific aortic stenosis is AVR or, in selected patients in the last years, an interventional treatment with TAVI. However, not all patients are good candidates for surgery and these results in a low number of operations compared to the prevalence of the disease.

As far as elderly patients are concerned, most series published in the last 10 years have reported operative mortality rates of approx. 10\% among the octogenarian population. Operative morbidity is also higher than in younger patients, and in particularthe frequency of stroke is reportedbetween $5 \%-10 \%$. Post-operative stroke is more frequent in patients who undergo associated coronary artery bypass grafting, most likely due to the impact of associated atherosclerosis [36]. Besides estimating mean operative mortality, risk assessment should be adapted to the patient's characteristics. Large series have shown a strong link between operative mortality and the following predictive factors:

- Advanced stage of heart disease (NYHA class IV, decreased left ventricular ejection fraction, or concomitant presence of a trial fibrillation).

- Comorbidities, in particular chronic obstructive pulmonary disease, renal insufficiency and diabetes.

- Need for urgent surgery.

- Associated atherosclerosis: significant coronary artery disease is present in approximately half of the patients over 75 years of age with AS, and they have a higher frequency of post-operative stroke which has an important impact on operative mortality.

Individual risk assessment should also take into consideration thepatient's complete clinical profile including, for example, porcelain aorta or prior radiation therapy. Multivariate scores, like The European System for Cardiac Operative Risk Evaluation (EuroSCORE), have been developed and validated to estimate operative mortality in cardiac surgery according to cardiac and non-cardiac characteristics. However, these scores have limitations when dealing with heart valve diseases in the elderly [37].

When assessing whether an octogenarian should undergo Aortic Valve Replacement (AVR), clinicians should not solely rely on the EuroSCORE, but should also take into account the degree of associated cardiomyopathy, severity of pulmonary hypertension and overall assessment of "frailty". The patient's willingness and desire for surgery also play a significant part in the overall decision, as do the experience and "track-record" of the surgical team.

Osswald et al. analysed 1545 consecutive patients with AS who underwent isolated surgical AVR between 1994 and 2006. Both additive and logistic EuroSCOREs were calculated for each patient and summed for expected 30-day mortality. Expected and observed mortalities were compared: 30-day mortality was low (34/1545, 2.2\%) and substantially overestimated by both additive $(6.1 \%)$ and logistic (9.3\%) EuroSCOREs. Although both EuroSCOREs stratified patients monotonically with respect to mortality risk, high-risk patients had a 3.6\% mortality rate (29/833), whereas additive and logistic EuroSCOREs predicted $8.3 \%$ and $14.8 \%$, respectively [38]. The more recent the era of surgery, the more pronounced the discrepancy between expected and observed mortalities was. Shanmugan et al. also observed that it is very hard to predict mortality for high-risk patients (with EuroSCORE $>$ of $18-20$ ). In this group, the perfect scoringsystem does not exist and therefore risk scores should not be taken out of context when counselling patients, regardless of their predictive accuracy. The reduced predictive ability of multivariate scores in elderly patients is probably related to the fact that high-risk groups only accounted for a small proportion of the populations for whom data were collected [38,39].

Life expectancy is most significantly influenced by comorbidities, which should be carefully looked for. Most works on TAVI point out the importance of assess patient's frailty for a better patient's selection. The term frailty is used to describe combinations of ageing, disease and other factors that make some people vulnerable. There is no broadly accepted definition or standard system for classification of elderly people who are at risk for adverse health outcomes. In addition to clinical evaluation, semi-quantitative scoring systems such as Fried Frailty Index, Columbia Frailty Index or Geriatric Status Scale (GSS) may be helpful. The Italian observational study on TAVI (OBSERVANT) is actually scoring patient on the basis of GSS [40]. After the post-operative period, late results of AVR are usually good in the elderly. 5-year survival rates are estimated at between $50 \%$ $70 \%$ after AVR in the octogenarian population. Of course, survival rates are lower than in younger patients, but they are favourable if compared with life expectancy in the general population of the same age. As compared to expected survival relative survival is especially good in the elderly. In fact, a comparison of patients who had been propensity-matched by risk profile (459 from each group) 
showed that the older group had a higher morbidity rate $(21.1 \%$ vs. $15.5 \% ; \mathrm{p}<0.03)$ but a similar mortality rate (5.2\% vs. $3.3 \% ; p=0.19)$ compared with the younger group [41]. Difficulties in decision making in this population are not only related to the decision of whether to operate or not, but also to the timing of surgery. Given the operative risk and the frequent reluctance of patients, surgery is seldom taken into consideration at the very beginning of symptoms. Symptoms are often difficult to interpret in the elderly and this also tends to delay surgery. AS-related symptoms often do not occur in the elderly when functional capability is limited by other reasons or when they are difficult to distinguish from concomitant cardiorespiratory illnesses. The Euro Heart Survey on valvular heart disease provided infor- mation on the current characteristics and management of patients with AS. Of the 216 patients aged $>75$ years who had severe symptomatic aortic AS, 72 (32\%) were denied surgery. Left ventricular dysfunction and older age were the two main characteristics associated with the decision not to operate (as assessed by multivariate analysis), whereas comorbidities, combined in the Charlson Co-morbidity Index (CSI), were not significantly associated with the decision of whether to operate or not [42-44].

Moreover, patients aged between 80 - 85 years with no major comorbidities and left ventricular ejection fraction between $30 \%-50 \%$ are still not referred for valve surgery because the risk/benefit ratio is not clear, even if these subjects would likely benefit from surgery in terms of duration and quality of life as compared with the spontaneous outcome of the disease. On the other hand, it is surprising that the CSI was not significantly associated with the decision not to operate. Most co-morbidities increase the operative risk and are also associated with a significant decrease in life expectancy, and therefore have a negative impact on the risk/benefit ratio of AVR [45-47].

\subsection{Balloon Aortic Valvuloplasty (BAV)}

Patients who are not eligible for AVR can be treated with valvuloplasty and potentially with TAVI, a relatively new procedure. Twenty years ago, Balloon Aortic Valvuloplasty (BAV) emerged as a promising alternative. However, the achieved results in aortic valve area and in aortic valve mean pressure gradient were lower than what was obtained by surgical AVR, and furthermore, balloon aortic valvuloplasty did not improve survival as compared with natural history. Moreover, the NHLBI (National Heart, Lung and Blood Institute) registry demonstrated high rates of acute complications with BAV. Procedural mortality was $3 \%$ and major complications occurred in up to $25 \%$ of patients [48]. This explains why both current American College of Cardiology and Ame- rican Heart Association guidelines restrict indications for BAV to situations in which it is used as a bridge for subsequent AVR. Furthermore, they also suggest that BAV might be a reasonable palliative treatment in elderly patients with aortic stenosis in whom surgical AVR cannot be performed because of serious co-morbid conditions. Don et al. compared procedural and in-hospital outcomes of BAV in patients below and above 80 years of age and found that those aged over 80 had smaller post-BAV aortic valve areas. Overall in-hospital mortality was $8.1 \%$ with non-significant differences between the two groups. Advanced age was not an independent predictor of in-hospital death, myocardial infarction, stroke, cardiac arrest or tamponade. The only difference was that patients over 80 years of age had a significantly higher incidence of intraprocedural intubation and cardiopulmonary resuscitation [48]. Moreover, after BAV there is a high restenosis rate, up to $80 \%$ in 1 year.

\subsection{Transcatheter Aortic Valve Implantation (TAVI)}

Percutaneous implantation of a transcatheter heart valve prosthesis now seems to offer the possibility of a chance in patients at high surgical risk or inoperable. For ethical reasons, the first cases were performed exclusively on patients who had contraindications to surgery because of end stage heart disease and/or severe comorbidities. This accounted for high mid-term mortality rates; however, it demonstrated the feasibility of this approach. Clinical criteria for select a patient for TAVI are illustrated in a common position statement from different European associations [49]. When the prosthesis cannot be delivered using an endovascular approach because of the peripheral artery status, it can be inserted using a minimally invasive, surgical approach. Alain Cribier described the first transcatheter aortic valve implantation in 2002 via an anterograde transeptal approach; afterwards the retrograde approach via femoral artery was developed. In the transfemoral procedureclose attention should be paid to the vascular access: after arterial puncture or surgical exposure, the femoral artery is dilated to accommodate the delivery sheath. BAV is performed under conditions of rapid pacing (200 beats/min) in order to minimize cardiac output and therefore minimize balloon movement upon inflation. When positioning is considered correct, the prosthesis is released: rapid pacing is used at this stage in balloon expandable but not in self-expanding devices. Depending on the size of thevalve, and consequently of the delivery sheath, closure of the vascular access can be effected surgically or usinga percutaneous closure device (Figure 3) [50].

In the transapical procedure access to the LV apex is 
gained through a left anterolateral mini-thoracotomy. The apex is punctured and the native valve is crossed anterogradely. After BAV, the valve prosthesis is implanted using a technique that is similar to the transfemoral approach. The LV apex is closed with a purse-string suture [51]. Two alternatives to the previously discussed access for patients with peripheral vasculopathy are: trans-sub- clavian approach (retrograde pathway that requires surgical exposure of the subclavian artery) and trans-aortic approach (retrograde approach that requires an upper "J-shape" mini-sternotomy); the presence of "porcelain" aorta and the risk of post-operative massive bleeding limit this approach to selected cases [52-54].

Prosthesis characteristics:

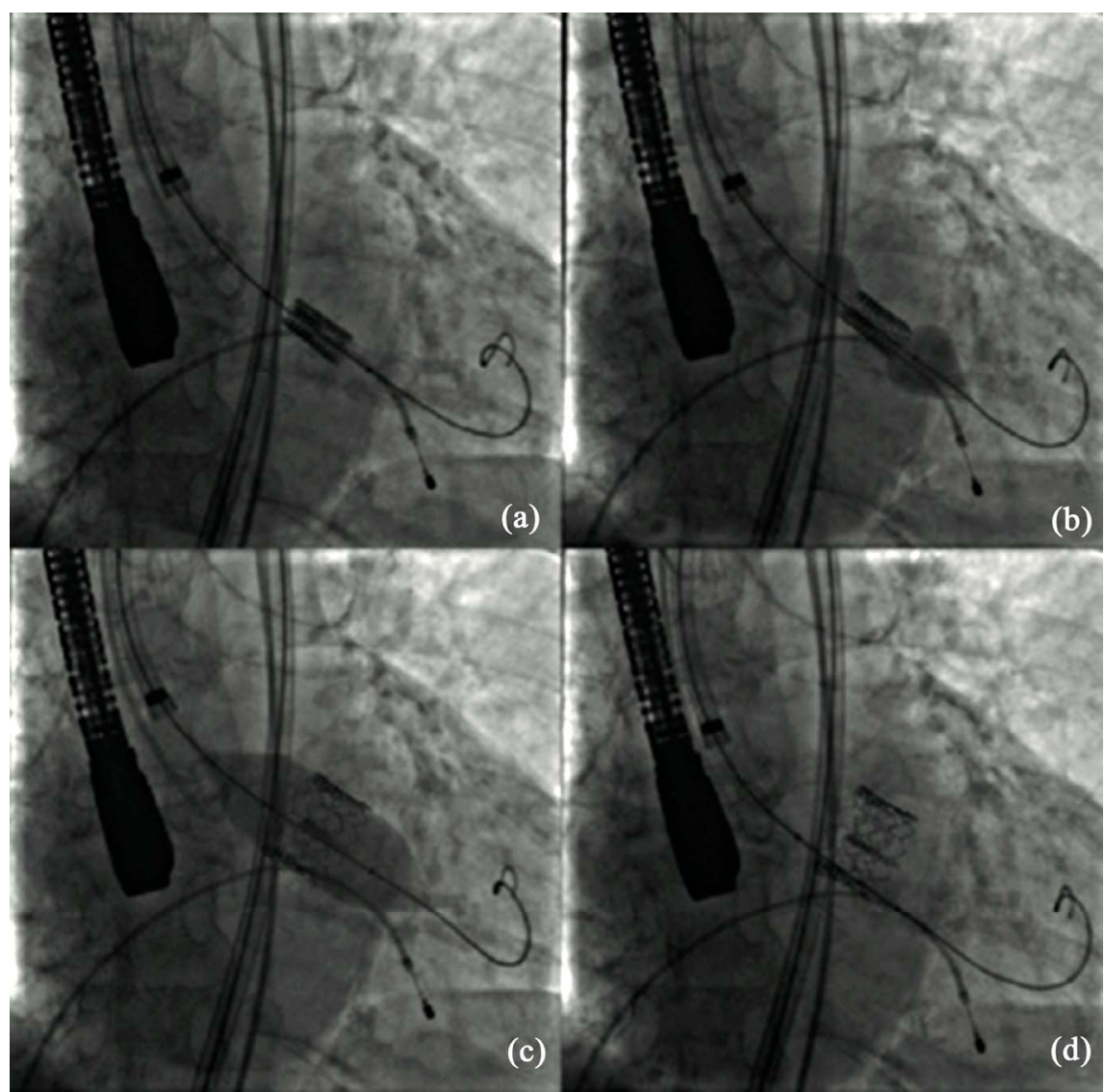

Figure 3. Phases of percutaneous heart valve replacement (TAVI). (a) Edwards heart valve is guided under fluoroscopy imaging into the correct position; (b) Balloon is inflated to expand the prosthetic valve; (c) Maximal balloon inflation for valve delivery; (d) Definitive valve positioning.

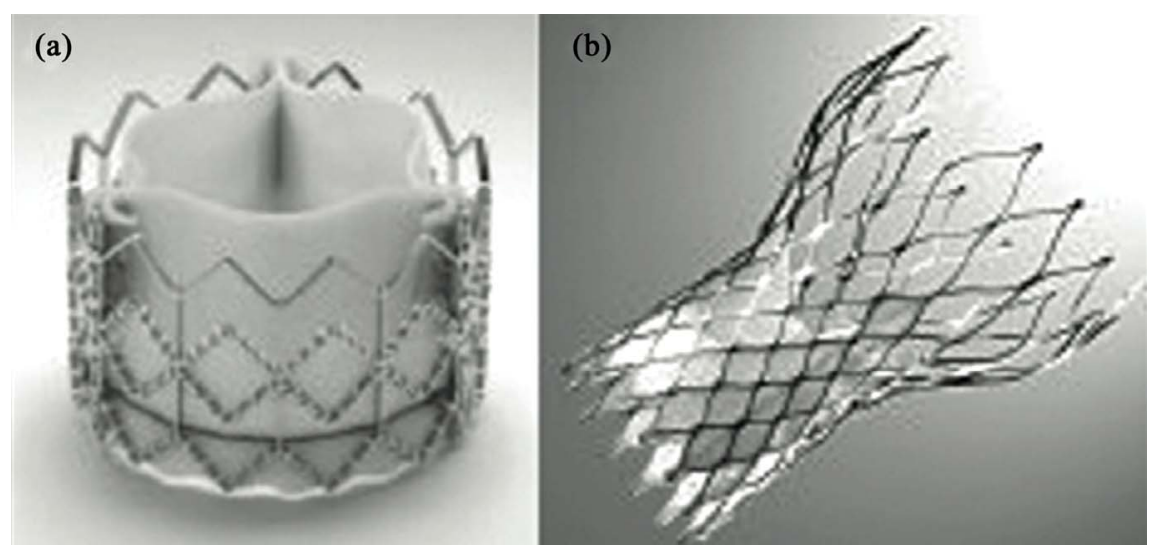

Figure 4. Valves used in percutaneous heart valve replacement (TAVI). (a) Edwards SAPIEN transcatheter heart valve (Edwards Lifesciences, Irvine, California); (b) Medtronic CoreValve aortic prosthesis (Medtronic, Minneapolis, MN). 
- Edwards SAPIEN Valve: balloon-mounted bovine pericardial valve within a stainless steel stent (Figure 4(a)). It is currently available in 2 sizes (23 and $26 \mathrm{~mm}$ ) for transfemoral and transapical approach, these valve diameters are suitable for implantation in aortic annulus diameters from 18 to $25 \mathrm{~mm}$. Furthermore there is a $29 \mathrm{~mm}$ prosthesis available only for transapical approach. Improvement in technology (mainly reduction in delivery profile) has increased the number of patients who are eligible for this kind of therapy and possibly reducing vascular complications [55].

- Medtronic CoreValve Revalving System: a porcine pericardial valve sewn within a self-expanding nitinol frame. It is currently available in diameters of $26 \mathrm{~mm}$, $29 \mathrm{~mm}$ and $31 \mathrm{~mm}$ to allow implantation in aortic valve annulus diameters up to $29 \mathrm{~mm}$ (Figure 4(b)). The delivery catheter diameter has also undergone generational changes to reduce the diameter from 25 French to the current 16 French that allows implantation in patients with iliac or sub-clavian artery diameters of $6 \mathrm{~mm}$ or more [56].

The only currently published, multi-centre, randomized trial regarding transcatheter valve implantation is the PARTNER trial (The Placement of Aortic transcatheter-valve Trial) which includes patients with severe, symptomatic aortic stenosis who are at high surgical risk (cohort A) or who are not candidates for surgery (cohort B). It compares Edwards SAPIEN Transcatheter Heart Valve implantation versus other surgical replacement valves (cohort A) and versus medical management and/or balloon aortic valvuloplasty (cohort B). The primary endpoint is freedom from death [57]. Cohort B of the PARTNER trial included 358 patients. Within the first 30 days, strokes and major vascular complications were found to be higher in the TAVI group, and the difference reached statistical significance for vascular complications. At one year, however, the rate of all-cause death was $30.7 \%$ in the TAVI group compared with $50.7 \%$ in the standardtherapy group. For those who survived 12 months, rates of NYHA class 3 or 4 heart failure were dramatically lower in the TAVI group [58]. Also results of PARTNER cohort A were positive. At 30 days, deaths were numerically lower in the TAVI group, but not statistically different $(3.4 \%$ in the transcatheter group and $6.5 \%$ in the surgical group; $\mathrm{P}=0.07$ ). By one year, deaths in both groups were nearly identical and met the predefined definition of non-inferiority ( $24.2 \%$ and $26.8 \%$ respectively; $\mathrm{P}=0.44$ ). More patients undergoing transcatheter replacement had an improvement in symptoms at 30 days, but by 1 year, there was not a significant between-group difference [59]. Over the last few years several observational registry studies have been published and data are now available. Data from German registry [60], Canadian registry [61], London study cohort [62] and French registry [63], show that short-term outcomes are usually good, resulting in successful implantation and 30-day survival rates medium of $94 \%$ and $89 \%$, respec- tively. In the Belgian registry one-month mortality was $12 \%$ for the Edwards and $11 \%$ for the CoreValve treated patients. One-year mortality was $22 \%$ in the CoreValve transfemoral treated patients, $0 \%$ in the CoreValve subclavian treated patients, $18 \%$ in the Edwards transfemoral treated patients and $37 \%$ in the Edwards transapical treated patients. These levels of mid-term mortality were mainly caused by age-related, non-cardiac complications [64]. Italian data on 633 patients who underwent TAVI with the third generation CoreValve device reported a procedural success of $98 \%$. The cumulative incidences of mortality were $5.4 \%$ at 30 days, $12.2 \%$ at 6 months, and $15.0 \%$ at 1 year. Clinical and hemodynamic benefits observed acutely after TAVI were sustained at 1 year [65]. With regards to two years follow-up: 126 patients underwent TAVI using the 18-F Medtronic CoreValve were examined. The overall technical success rate was $83.1 \%$ and thirty-day all-cause mortality was $15.2 \%$ whereas at 2 years was $38.1 \%$. Hemodynamic results remained unchanged during follow-up, functional class improved in $80 \%$ of patients and remained stable over time and there was no incidence of structural valve deterioration [66]. Two Danish trials are now on-going. The aim of the first trial is to compare TAVI vs. SAVR in patients $>70$ years with Medtronic valves, and in particular with regard to long term outcome (5 year follow up) [67]. The second will analyse the same problem with the SAPIEN valve and will study early outcome [68].

\section{Impact of TAVI in Elderly Subjects with Aortic Stenosis}

Data from more than 30 developed countries showed that in 1950 , the probability of survival from 80 years of age to 90 years of age was on average $15 \%-16 \%$ for women and $12 \%$ for men. In 2002 , these figureshad reached $37 \%$ and $25 \%$, respectively. With few exceptions, mortality generally keeps falling in all selected countries for both genders and at both ages. Nowadays, a 90 - 94-year-old woman in Italy has a life expectancy of 4 years. TAVI will improve life expectancy in those elderly in which traditional surgery will be contraindicated due to high aged surgical risk and complex comorbidities. TAVI will require a multidisciplinary team that includes surgeons, cardiologists, geriatricians, interventional cardiologists and anaesthesiologists because the non-invasive treatment has to be a personalized decision (based on scores, but mainly on an overall evaluation of patient).

In an elderly population, mortality risk and quality of life considerations are equally important to define the final therapeutic option. One fifth of the patients who 
were discharged after traditional surgery experienced a worsening of their quality of life and some lost their independence. Moreover, even successful valve replacement does not prevent mortality that is attributable to the patient's comorbidities. Maximum survival must be balanced by the maximum level of satisfaction and general well-being over years of survival. In octogenarians, symptom relief and improvement in quality of life should be of greater importance than increased life expectancy. The TAVI approach in older people will solve the aortic disease with less risk at surgery maintaining (or regaining) as much personal independence as possible $[57,69]$.

\section{Conclusion}

Aortic stenosis is the most common valvular disease in the elderly. It is associated with very poor prognosis in the absence of surgical treatment and very bad quality of life. The high frequency of AS combined with the increasing expected lifespan has generated a new category of complex elderly patients with AS who are referred to cardiac surgery. The new disposable therapeutic options, ranging from valvuloplasty to TAVI, highlights the need of a team approach involving clinicians and surgeons to better define the best surgical options in the every elderly patient.

\section{REFERENCES}

[1] J. Walston, E. C. Hadley, L. Ferrucci, J. M. Guralnik, A. B. Newman, S. A. Studenski, et al., "Research Agenda for Frailty in Older Adults: Toward a Better Understanding of Physiology and Etiology: Summary from the American Geriatrics Society/National Institute on Aging Research Conference on Frailty in Older Adults," Journal of the American Geriatrics Society, Vol. 54, No. 6, 2006, pp. 991-1001. doi:10.1111/j.1532-5415.2006.00745.x

[2] Hurst, "Il Cuore," McGraw Hill Companies, New York, 1987-1999.

[3] B. A. Carabello and W. J. Paulus, "Aorticstenosis," Lancet, Vol. 373, No. 9667, 2009, pp. 956-966. doi:10.1016/S0140-6736(09)60211-7

[4] L. P. Fried, N. O. Borhani, P. Enright, C. D. Furberg, J. M. Gardin, R. A. Kronmal, et al., "The Cardiovascular Health Study: Design and Rationale," Annals of Epidemiology, Vol. 1, No. 3, 1991, pp. 263-276. doi:10.1016/1047-2797(91)90005-W

[5] W. S. Aronow and I. Kronzon, "Prevalence and Severity of Valvular Aortic Stenosis Determinated by Doppler Echocardiography and Its Association with Echocardiographic and Electrocardiographic Left Ventricular Hypertrophy and Physical Signs of Aortic Stenosis in Elderly Patients," American Journal of Cardiology, Vol. 67, No. 8, 1991, pp. 776-777. doi:10.1016/0002-9149(91)90542-S

[6] P. G. Supino, J. S. Borer, J. Preibisz and A. Bornstein, "The Epidemiology of Valvular Heart Disease: A Growing Public Health Problem," Heart Failure Clinics, Vol. 2, No. 4,
2006, pp. 379-393. doi:10.1016/j.hfc.2006.09.010

[7] R. Shueler, C. Hammerstingl, J. M. Sinning, G. Nickenig and H. Omran, "Prognosis of Octogenarians with Severe Aortic Valve Stenosis at High Risk for Cardiovascular Surgery," Heart, Vol. 96, No. 22, 2010, pp. 1831-1836. doi:10.1136/hrt.2010.202663

[8] A. Parolari, C. Loardi, L. Mussoni, L. Cavallotti, M. Camera, P. Biglioli, et al., "Nonrheumatic Calcific Aortic Stenosis: An Overview from Basic Science to Pharmacological Prevention," European Journal Cardio-Thoracic Surgery, Vol. 35, No. 3, 2009, pp. 493-504. doi:10.1016/j.ejcts.2008.11.033

[9] N. Rajamannan, "Calcific Aortic Stenosis: Lessons Learned from Experimental and Clinical Studies," Arteriosclerosis, Thrombosis, and Vascular Biology, Vol. 29, No. 2, 2009, pp. $162-168$.

[10] R. Capoulade, M. A. Clavel, J. G. Dumesnil, et al., "Astronomer Investigators. Impact of Metabolic Syndrome on Progression of Aortic Stenosis: Influence of Age and Statin Therapy," Journal of the American College of Cardiology, Vol. 60, No. 3, 2012, pp. 216-223. doi:10.1016/j.jacc.2012.03.052

[11] N. Rajamannan and C. Otto, "Targeted Therapy to Prevent Progression of Calcific Aortic Stenosis," Circulation, Vol. 110, No. 10, 2004, pp. 1180-1182. doi:10.1161/01.CIR.0000140722.85490.EA

[12] J. W. Kennedy, R. D. Twiss, J. R. Blackmon and H. T. Dodge, "Quantitative Angiocardiography. Relationships on Left Ventricular Pressure, Volume and Mass in Aortic Valve Disease," Circulation, Vol. 38, No. 5, 1968, pp. 838845. doi:10.1161/01.CIR.38.5.838

[13] W. P. Hood, C. E. Rackley and E. L. Rolett, "Wall Stress in the Normal and Hypertrophied Human Left Ventricle," American Journal of Cardiology, Vol. 22, No. 4, 1968, pp. 550-558. doi:10.1016/0002-9149(68)90161-6

[14] O. M. Hess, B. Villari and H. P. Krayenbuehl, "Diastolic Dysfunction in Aortic Stenosis," Circulation, Vol. 87, No. 5, 1993, pp. 73-76.

[15] Ross Jr., "Afterload Mismatch in Aortic and Mitral Valve Disease: Implications for Surgical Therapy," Journal of the American College of Cardiology, Vol. 5, No. 4, 1985, pp. 811-826. doi:10.1016/S0735-1097(85)80418-6

[16] G. R. Green and D. C. Miller, "Continuing Dilemmas Concerning Aortic Valve Replacement in Patients with Advanced Left Ventricular Systolic Dysfunction," Journal of Heart Valve Diseases, Vol. 6, No. 6, 1997, pp. 562-579.

[17] F. Antonini-Canterin and G. L. Nicolosi, "Hemodynamic Progression of Aortic Valve Stenosis in the Adult: Implications of the Clinical Management of Asymptomatic Patients," Italian Heart Journal, Vol. 3, No. 5, 2002, pp. 478485.

[18] J. Ross and E. Braunwald, "Aortic Stenosis," Circulation, Vol. 38, 1968, pp. 61-67. doi:10.1161/01.CIR.38.1S5.V-61

[19] E. Braunwald, "On the Natural History of Severe Aortic Stenosis," Journal of the American College of Cardiology, Vol. 15, No. 5, 1990, pp. 1018-1020. doi:10.1016/0735-1097(90)90235-H 
[20] J. F. Malouf, M. Enriquez-Sarano, P. A. Pellikka, J. K. Oh, K. R. Bailey, K. Chandrasekaran, et al., "Severe Pulmonary Hypertension in Patients with Severe Aortic Valve Stenosis: Clinical Profile and Prognostic Implications," American Journal of Cardiology, Vol. 40, No. 4, 2002, pp. 789-795. doi:10.1016/S0735-1097(02)02002-8

[21] R. V. Shah, N. R. Desi and P. T. O'Gara, "Asymptomatic Severe Aortic Stenosis: Silence of the Lambs," Circulation, Vol. 122, No. 17, 2010, pp. 1734-1739. doi:10.1161/CIRCULATIONAHA.110.989152

[22] J. Turina, O. Hess, F. Sepulcri and H. P. Krayenbuehl, "Spontaneous Course of Aortic Valve Disease," European Heart Journal, Vol. 8, No. 5, 1987, pp. 471-483.

[23] P. A Pellikka, M. E. Sarano, R. A. Nishimura, J. F. Malouf, K. R. Bailey, C. G. Scott, et al., "Outcome of 622 Adults with Asymptomatic, Hemodynamically Significant Aortic Stenosis during Prolonged Follow-Up," Circulation, Vol. 111, No. 24, 2005, pp. 3290-3295.

[24] R. Rosenhek, R. Zilberszac, M. Schemper, M. Czerny, G. Mundigler, S. Graf, et al., "Natural History of Very Severe Aortic Stenosis," Circulation, Vol. 121, No. 1, 2010, pp. 151-156. doi:10.1161/CIRCULATIONAHA.109.894170

[25] J. Stritzke, P. Linsel-Nitschke, M. R. P. Markus, B. Mayer, W. Lieb, A. Luchner, et al., "Association between Degenerative Aortic Valve Disease and Long-Term Exposure to Cardiovascular Risk Factors: Results of the Longitudinal Population-Based KORA/MONICA Survey," European Heart Journal, Vol. 30, No. 16, 2009, pp. 2044-2053. doi:10.1093/eurheartj/ehp287

[26] M. Peltier, F. Trojette, M. E. Sarano, F. Grigioni, M. Slama and C. M. Tribouilloy, "Relation between Cardiovascular Risk Factors and Nonrheumatic Severe Calcific Aortic Stenosis among Patients with a Three-Cuspid Aortic Valve," American Journal of Cardiology, Vol. 91, No. 1, 2003, pp. 97-99. doi:10.1016/S0002-9149(02)03010-2

[27] C. M Otto, I. G. Burwash, M. E. Legget, B. I. Munt, M. Fujioka, N. L. Healy, et al., "Prospective Study of Asymptomatic Valvular Aortic Stenosis: Clinical, Echocardiographic, and Exercise Predictors of Outcome," Circulation, Vol. 95, No. 9, 1997, pp. 2262-2270. doi:10.1161/01.CIR.95.9.2262

[28] A. Vahanian, H. Baumgartner, E. Butchart, et al., "Guidelines on the Management of Valvular Heart Disease. The Task Force on the Management of Valvular Heart Disease of the European Society of Cardiology," European Heart Journal, Vol. 28, No. 2, 2007, pp. 230-268.

[29] S. Rispler, D. Rinkevich and W. Markiewicz, "Missed Diagnosis of Severe Symptomatic Aortic Stenosis," American Journal of Cardiology, Vol. 76, No. 10, 1995, pp. 728-730. doi:10.1016/S0002-9149(99)80210-0

[30] P. C. Come, M. F. Riley, R. G. McKay, et al., Echocardiographic Assessment of Aortic Valve Area in Elderly Patients with Aortic Stenosis and of Changes of Valve Area after Percutaneous Balloon Valvuloplasty," Journal of the American College of Cardiology, Vol. 10, No. 1, 1987, pp. 115-124. doi:10.1016/S0735-1097(87)80169-9

[31] P. Teirstein, M. Yeager, P. G. YocK and R. L. Popp, "Doppler Echocardiographic Measurements of Aortic Valve Area in Aortic Stenosis: A Non-Invasive Application of the Gorlin Formula," American Journal of Cardiology, Vol. 8, No. 5, 1986, pp. 1059-1065. doi:10.1016/S0735-1097(86)80382-5

[32] J. Zamorano, L. Badano, C. Bruce, K. L. Chan, A. Goncalves, R. T. Hahn, et al., "EAE/ASE Recommendations for the Use of Echocardiography in the New Transcatheter Interventions for Valvular Heart Disease," European Heart Journal, Vol. 32, No. 17, 2011, pp. 2189-2214. doi:10.1093/eurheartj/ehr259

[33] A. Galan, W. A. Zoghbi and M. A. Quinones, "Determination of Severity of Valvular Aortic Stenosis by Doppler Echocardiography and Relation of Findings to Clinical Outcome and Agreement with Hemodynamic Measurements Determined at Cardiac Catheterization," American Journal of Cardiology, Vol. 67, No. 11, 1991, pp. 10071012. doi:10.1016/0002-9149(91)90175-K

[34] J. Bermejo, M. A. García-Fernández, E. G. Torrecilla, H. Bueno, M. M. Moreno, D. S. Román, et al., "Effects of Dobutamine on Doppler Echocardiographic Indexes of Aortic Stenosis," Journal of the American College of Cardiology, Vol. 28, No. 5, 1996, pp. 1206-1213. doi:10.1016/S0735-1097(96)00287-2

[35] L. F. Tops, D. A. Wood, V. Delgado, J. D. Schuijf, J. R. Mayo, S. Pasupati, et al., "Noninvasive Evaluation of the Aortic Root with Multislice Computed Tomography: Implications for Transcatheter Aortic Valve Replacement," Journal of the American College of Cardiology, Vol. 1, No. 3, 2008, pp. 321-330.

[36] I. Logeais, T. Langanay, R. Roussin, A. Leguerrier, C. Rioux, J. Chaperon, et al., "Surgery for Aortic Stenosis in Elderly Patients. A Study of Surgical Risk and Predictive Factors," Circulation, Vol. 90, No. 6, 1994, pp. 2891-2898. doi:10.1161/01.CIR.90.6.2891

[37] D. Wendt, B. R. Osswald, K. Kayser, M. Thielmann, P. Tossios, P. Massoudy, et al., "Society of Thoracic Surgeons Score Is Superior to the EuroSCORE Determining Mortality in High Risk Patients Undergoing Isolated Aortic Valve Replacement," The Annals of Thoracic Surgery, Vol. 88, No. 2, 2009, pp. 468-474. doi:10.1016/j.athoracsur.2009.04.059

[38] B. R. Osswald, V. Gegouskov, D. Badowski-Zyla, U. Tochtermann, G. Thomas, S. Hagl, et al., "Overstimation of Aortic Valve Replacement Risk by EuroSCORE: Implications for Percutaneous Valve Replacement," European Heart Journal, Vol. 30, No. 1, 2009, pp. 74-80. doi:10.1093/eurheartj/ehn523

[39] G. Shanmugam, M. West and G. Berg, "Additive and Logistic EuroSCORE Performance in High Risk Patients," Interactive Cardio Vascular Thoracic Surgery, Vol. 4, No. 4, 2005, pp. 299-303. doi:10.1510/icvts.2004.104042

[40] Gruppo di Lavoro Tecnico dello Studio Observant, "Observant: Studio Osservazionale per la Valutazione di Appropriatezza, Efficienza ed Efficacia Delle Procedure AVR-TAVI nel Trattamento Della Stenosi Aortica Sintomatica Severa. Protocollo di Studio," Giornale Italiano di Cardiologia, Vol. 11, No. 12, 2010, pp. 897-909.

[41] F. Roques, S. A. Nashef and P. Michel, "EuroSCORE Study Group. Risk Factors for Early Mortality after Valve Surgery in Europe in the 1990's: Lesson from the Euro- 
score Pilot Program," Journal of Heart Valve Diseases, Vol. 10, 2001, pp. 572-578.

[42] B. Iung, G. Baron, E. G. Butchart, F. Delahaye, C. GohlkeBärwolf, O. W. Levang, et al., "A Prospective Survey of Patients with Valvular Heart Disease in Europe: The Euro Heart Survey on Valvular Heart Disease," European Heart Journal, Vol. 24, No. 13, 2003, pp. 1231-1243. doi:10.1016/S0195-668X(03)00201-X

[43] B. Iung, A. Cachier, G. Baron, D. Messika-Zeitoun, F. Delahaye, P. Tornos, et al., "Decision Making in Elderly Patients with Severe Aortic Stenosis: Why Are So Many Denied Surgery," European Heart Journal, Vol. 26, No. 24, 2005, pp. 2714-2720. doi:10.1093/eurheartj/ehi471

[44] B. Iung, "Management of the Elderly Patient with Aortic Stenosis," Heart, Vol. 94, No. 4, 2008, pp. 519-524. doi:10.1136/hrt.2007.122804

[45] F. G. Bakaeen, D. Chu, M. Ratcliffe, R. R. Gopaldas, A. S. Blaustein, R. Venkat, et al., "Severe Aortic Stenosis in a Veteran Population: Treatment Considerations and Survival," The Annals of Thoracic Surgery, Vol. 89, No. 2, 2010, pp. 453-458. doi:10.1016/j.athoracsur.2009.10.033

[46] F. G. Bakaeen, D. Chu, J. Huh and B. A. Carabello, "Is an Age of 80 Years or Greater an Important Predictor of Short-Term Outcomes of Isolated Aortic Valve Replacement in Veterans," The Annals of Thoracic Surgery, Vol. 90, No. 3, 2010, pp. 769-774.

doi:10.1016/j.athoracsur.2010.04.066

[47] P. Kvidal, M. Bergstrom, L. G. Horte and E. Sthale, "Observed and Relative Survival after Aortic Valve Replacement," Journal of the American College of Cardiology, Vol. 35, No. 3, 2000, pp. 747-756. doi:10.1016/S0735-1097(99)00584-7

[48] Nhlbi Balloon Valvuloplasty Registry Participants, "Percutaneous Balloon Aortic valvuloplasty. Acute and 30Day Follow up Results in 674 Patients from the NHLBI Balloon Valvuloplasty Registry," Circulation, Vol. 84, 1991, pp. 2383-2397. doi:10.1161/01.CIR.84.6.2383

[49] C. W. Don, C. Witzke, R. J. Cubeddu, J. Herrero-Garibi, E. Pomerantsev, A. E. Caldera, et al., "Comparison of Procedural and in Hospital Outcomes of Percutaneous Balloon Aortic Valvuloplasty in Patients $>80$ Years versus Patients $<80$ Years," American Journal of Cardiology, Vol. 105, No. 12, 2010, pp. 1815-1820. doi:10.1016/j.amjcard.2010.01.366

[50] A. Vahanian, O. Alfieri, N. Al-Attar, M. Antunes, J. Bax, B. Cormier, et al., "Transcatheter Valve Implantation for Patients with Aortic Stenosis: A Position Statement from the European Association of Cardio Thoracic Surgery (EACTS) and the European Society of Cardiology (ESC), in Collaboration with the European Association of Percutaneous Cardiovascular Interventions (EAPCI)," European Heart Journal, Vol. 29, No. 11, 2008, pp. 1463-1470. doi:10.1093/eurheartj/ehn183

[51] A. Cribier, H. Eltchaninoff, A. Bash, N. Borenstein, C. Tron, F. Bauer, et al., "Percutaneous Transcatheter Implantation of an Aortic Valve Prosthesis for Calcific Aortic Stenosis: First Human Case Description," Circulation, Vol. 106, No. 24, 2002, pp. 3006-3008. doi:10.1161/01.CIR.0000047200.36165.B8
[52] T. Walther, T. Dewey, M. A. Borger, J. Kempfert, A. Linke, R. Becht, et al., "Transapical Aortic Valve Implantation: Step by Step," The Annals of Thoracic Surgery, Vol. 87, No. 1, 2009, pp. 276-283. doi:10.1016/j.athoracsur.2008.08.017

[53] A. S. Petronio, M. De Carlo, F. Bedogni, A. Marzocchi, S. Klugmann, F. Maisano, et al., "Safety and Efficacy of the Subclavian Approach for Transcatheter Aortic Valve Implantation with the Core Valve Revalving System," Circulation: Cardiovascular Interventions, Vol. 3, No. 4, 2010, pp. 359-366.

[54] G. Latsios, U. Gerckens and E. Grube, "Transaortic Transcatheter Aortic Valve Implantation: A Novel Approach for the Truly 'No-Access Option' Patients," Catheterization and Cardiovascular Interventions, Vol. 75, No. 7, 2010, pp. 1129-1136.

[55] F. Nietlispach, N. Wijesinghe, D. Wood, R. G. Carere and J. G. Webb, "Current Balloon-Expandable Transcatheter Heart Valve and Delivery System," Catheterization and Cardiovascular Interventions, Vol. 75, No. 2, 2010, pp. 295300. doi:10.1002/ccd.22279

[56] E. Grube, L. Buellesfeld, R. Mueller, B. Sauren, B. Zickmann, D. Nair, et al., "Progress and Current Status of Percutaneous Aortic Valve Replacement: Results of Three Device Generation of the Core Valve Revalving System," Catheterization and Cardiovascular Interventions, Vol. 1, No. 3, 2008, pp. 167-175.

[57] M. B. Leon, "The Partner Trial: Placement of Aortic Transcatheter Valve Trial," Clinical Trials Gov, 2010.

[58] M. B. Leon, C. R. Smith, M. Mack, D. C. Miller, J. W. Moses, L. G. Svensson, et al., "Transcatheter Aortic Valve Implantation for Aortic Stenosis in Patients Who Cannot Undergo Surgery," The New England Journal of Medicine, Vol. 363, No. 17, 2010, pp. 1597-1607. doi:10.1056/NEJMoa1008232

[59] C. R. Smith, M. B. Leon, M. J. Mack, D. C. Miller, J. W. Moses, L. G. Svensson, et al., "Transcatheter versus Surgical Aortic-Valve Replacement in High-Risk Patients,' The New England Journal of Medicine, Vol. 364, No. 23, 2011, pp. 2187-2198. doi:10.1056/NEJMoa1103510

[60] R. Zahn, U. Gerckens, E. Grube, A. Linke, H. Sievert, H. Eggebrecht, et al., "Transcatheter Aortic Valve Implantation: First Results from a Multi-Centre Real Word Registry," European Heart Journal, Vol. 32, No. 2, 2011, pp. 198-204. doi:10.1093/eurheartj/ehq339

[61] J. Rodés-Cabau, J. G. Webb, A. Cheung, J. Ye, E. Dumont, C. M. Feindel, et al., "Transcatheter Aortic Valve Implantation for the Treatment of Severe Symptomatic Aortic Stenosis in Patients at Very High or Prohibitive Surgical Risk: Acute and Late Outcomes of the Multicentre Canadian Experience," Journal of the American College of Cardiology, Vol. 55, No. 11, 2010, pp. 10801090. doi:10.1016/j.jacc.2009.12.014

[62] R. Dworakowski, P. A. MacCarthy, M. Monaghan, S. Redwood, A. El-Gamel, C. Young, et al., "Transcatheter Aortic Valve Implantation for Severe Aortic Stenosis-A New Paradigm for Multidisciplinary Intervention: A Prospective Cohort Study," American Heart Journal, Vol. 160, No. 2, 2010, pp. 237-243. 
doi:10.1016/j.ahj.2010.05.014

[63] H. Eltchaninoff, A. Prat, M. Gilard, A. Leguerrier, D. Blanchard, G. Fournial, et al., "Transcatheter Aortic Valve Implantation: Early Results of the France Registry," European Heart Journal, Vol. 32, No. 2, 2011, pp. 191-197. doi:10.1093/eurheartj/ehq261

[64] J. M. Bosmans, J. Kefer, B. De Bruyne, P. Herijgers, C. Dubois, V. Legrand, et al., "Procedural, 30-Day and One Year Outcome Following CoreValve or Edwards Transcatheter Aortic Valve Implantation: Results of the Belgian National Registry," Interactive Cardiovascular and Thoracic Surgery, Vol. 12, No. 5, 2011, pp. 762-767. doi:10.1510/icvts.2010.253773

[65] C. Tamburino, D. Capodanno, A. Ramondo, A. S. Petronio, F. Ettori, G. Santoro, et al., "Incidence and Predictors of Early and Late Mortality after Transcatheter Aortic Valve Implantation in 633 Patients with Severe Aortic Stenosis," Circulation, Vol. 123, No. 3, 2011, pp. 299-308. doi:10.1161/CIRCULATIONAHA.110.946533

[66] L. Buellesfeld, U. Gerckens, G. Schuler, R. Bonan, J. Ko- vac, P. W. Serruys, et al., "2-Year Follow-Up of Patients Undergoing Transcatheter Aortic Valve Implantation Using a Self-Expanding Valve Prosthesis," Journal of the American College of Cardiology, Vol. 57, No. 16, 2011, pp. 1650-1657. doi:10.1016/i.jacc.2010.11.044

[67] D. A. Steinbrüchel, "Transcatheter Compared to Surgical Valve Implantation in Patients with Severe Aortic Valve Stenosis (TAVIvsSAVR)," Clinical Trials Gov, 2010.

[68] L. Thuesen, "Transapical Transcatheter Treatment versus Conventional Surgery in Patients with Native Aortic Valve Stenosis," Clinical Trials Gov, 2009.

[69] M. A. Clavel, J. G. Webb, J. Rodés-Cabau, J. B. Masson, E. Dumont, R. De Larochellière, et al., "Comparison Between Transcatheter and Surgical Prosthetic Valve Implantation in Patients with Severe Aortic Stenosis and Reduced Left Ventricular Ejection Fraction," Circulation, Vol. 122, No. 19, 2010, pp. 1928-1936. doi:10.1161/CIRCULATIONAHA.109.929893 